\title{
Naturaleza y poesía en Sandro Penna
}

\author{
Carmelo Vera Saura
}

El artista necesita expresar sus sentimientos a través de los elementos que componen la naturaleza, que servirá de trasfondo, de indicio o de símbolo de su interioridad. Recurre siempre a la naturaleza y su variedad de fenómenos y elementos para tomarlos como punto de comparación física o espiritual, metonímica o metafórica, del objeto de su discurso poético. La creación artística necesita de la representación de la naturaleza no sólo como mímesis sino como su otro con el que dialogar.

Pero es el poeta quien concibe los elementos naturales con una función específica para expresar su sentimiento o visión del mundo. El discurso poético se enuclea mediante un diálogo real o tácito entre yo-tú (él). En ausencia del tú amado o en la imposibilidad de un diálogo con él'se transfiere al orden natural cuanto es negado y oculto. El poeta dialoga con la naturaleza y, por tanto, necesita antropomorfizar el mundo, dotarlo de animación. El hombre no puede conocer ni concebir otro mundo sin darle una forma humana. Esta antropomorfización es una búsqueda de la otredad, del otro como relación en el mundo. Cuando ese tú-otro se desea cancelar el inconsciente se encarga de desvelarlo.

En la poesía de Sandro Penna la persona amada se proyecta fusionándose con la naturaleza en cuyo ámbito arquetípico superará el espacio y el tiempo a la vez que adquieren los elementos naturales forma humana tras recibir el soplo vivificante del amor o simplemente para crear la otredad dialogante. La animación de los elementos naturales se presenta en la poesía de Sandro Penna unida al sentimiento amoroso, fuerza principal que hace a aquéllos partícipes de su emotividad. Esta animación amorosa de la poesía penniana se manifiesta con muy diferentes características, pero es en muchas ocasiones el amor el hálito mediador entre poeta y naturaleza. Cassirer habla de la simpattia del tutto: "In realtà la dottrina stoica di un pneuma onnipervadente, di un soffio diffuso in tutto l'universo che dà ad ogni cosa la forza che la tienen insieme, presenta analogie con certi concetti primitivi, col mana dei polinesiani, col wakan del sioux» ${ }^{1}$.

\footnotetext{
${ }^{1}$ Ernst Cassirer, Saggio sull'uomo, Roma, Armando, 1982, p. 181.
} 
El amor es la fuerza oculta de la naturaleza que puede armonizar al hombre con el mundo para darle un sentido liberador más allá de las constricciones humanas. El amor es la única virtud natural para Penna capaz de elevar al hombre del estado de miseria y disgregación en que vive: «Viva restal la dolce persuasione di una fitta/rete d'amore ad inquietare il mondo", "Il mondo che Vi pare di cateneltutto è tessuto d'armonie profonde»".

Es interesante poner en relación el narcisismo amoroso penniano ${ }^{3}$ con la figurativización de algún elemento como es el caso del lago: «Nel chiuso lago, sola, senza vento/la mia nave trascorre, ad ora ad ora./Fremono i fiori sotto i ponti. Sentol la mia tristezza accendersi ancora». El lago se caracteriza doblemente por figurativizar el narcisismo, primeramente porque sus aguas quietas son siempre propicias para que se refleje el rostro de Narciso enamorado, y, por otra parte, su forma cerrada coadyuva a potenciar la forma incomunicante y aislada del amor narcisista. La imagen del lago le sirve, pues, para dar forma a esa vida-deseo (nave que transcurre) que no encuentra comunión sino en su propia reflexividad, es decir, en las aguas del lago, imagen de ensoñación narcisista ${ }^{4}$ ante la ausencia del objeto de deseo. Igualmente las flores se antropomorfizan y se narcisizan pues son el correlato del estado sentimental explicitado en la tristeza del último verso al tiempo que se contemplan en las aguas del lago.

De los cuatro elementos de la naturaleza es el mar el que propicia la liberación eufórica de su onirismo y de sus instintos inconscientes. La ensoñación de la imagen sosegada del mar caracterizada por los semas /frescor/ y /sosiego/ figurativiza la liberación del imaginario social y la pulsión del objeto de deseo, el marinero: « $e$ vicino a me la figura di un marinaiole un mare tutto fresco di colore », «Il mare è tutto azzurro.I

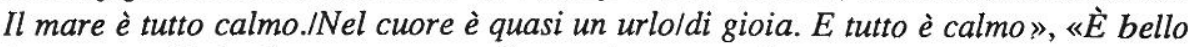
lavorare/nel buio di una stanza/con la testa in vacanzallungo un azzurro mare ». En el último ejemplo la oposición oscuridad/color (buio-azzurro) expresa la oposición inhibición/liberación. Luz y agua euforizan el encuentro del muchacho amado: «Ecco il fanciullo acquatico e felice./Ecco il fanciullo gravido di luce.../Dolce stagione di silenzio e di sole...». El agua es el lugar del nacimiento de la figura adolescente, $\mathrm{y}$, por tanto, de la ensoñación del deseo: «Il mio Amore era nudolin riva di un mare sonoro». El poeta intenta a través de la iteración fónica la fijación de la ensoñación material. Se puede decir que la aliteración de $/ \mathrm{m} /$ (mio-Amore-mare) y la paronomasia vocal+R+vocal (AmORE-ERA-mARE-sonORO) de grupos fónicos, fenómenos cuyo porcentaje es alto en la poesía penniana, se constituyen en sustitutos inconscientes que reconocen en su identidad material el objeto de deseo: «In questo caso, il poetico parlerebbel' inconscio

\footnotetext{
${ }^{2}$ Todos los poemas se citan de Tutte le poesie, Milano, Garzanti, 1970.

${ }^{3}$ Cfr. Carmelo Vera, «Eros narcisista en la poesía de Sandro Penna», Estudios Románicos, Homenaje al profesor Luis Rubio II, Universidad de Murcia, vol. 5, 1987-88-89, pp. 1403-1417.

${ }^{4}$ Es sabida la relación que establece Gaston Bacherlard entre las aguas y la ensoñación narcisista aludiendo al propio mito de Narciso en El agua y los suefios, México, F.C.E., 1978 (1942 ${ }^{1}$ ), pp. 39-55. Rosario Scrimieri individúa la imagen narcisista del lago en la poesía de Vittorio Sereni, en algunos poemas de Frontiera, 1933, como Inverno, en El universo poético de Vittorio Sereni, Madrid, Universidad Complutense, 1987, pp. 143-166.
} 
prima dell' interdizione (prima della parola analitica), lo direbbe como desiderio libero e senza oggetto (senza concetto), lo assumerebbe (lo costituerebbe) come motilità, ritmicità, fonalità pure, anteriori ai sistemi di significato»s.

Este lugar privilegiado se convierte en mítico, en arquetipo de la quietud, de la totalidad y en reino de la euforia del poeta: «Il mare è tutto azzurro./Il mare è tutto calmo./Nel cuore è quasi unurlo/di gioia. E tutto è calmo». La variaciónléxica y sintáctica es mínima para remarcar tres rasgos, /color/, /quietud/y /euforia/, en una gradación de lo externo concreto (mare), al interno del yo (cuore) y de ahía lo externo cósmico (tutto).

El agua, junto al sol, va unida a la ensoñación del objeto amoroso y de la fecundidad del amor: «Piove sulla città. Piove sul campo,/ove incontrai, nel sole, il lieto amico.//Ma innocenti/peccati in me la pioggia riaccende». "Piovve nel nostro amore ardentemente/tutta l' estate $»^{6}$, pero no posee un carácter visual sino sensorial, por lo que va asociada al ansia erótica, seabajo la figuratización de la fuente o delmar: «Se trasaliscel il fermo getto/della fontana,/la tramontana/curva il mio cuore/verso un ardore/già maledetto», «Com' era l' onda sullo scoglio aperta/cosi su quella fronte a me diletta/era il mio amore -e non sapevo quanto/ne gioisse lo scoglio o fosse in pianto». El deseo figurativizado en la imagen arquetípica del agua responde a un carácter dinámico y de totalidad, de integración en un elemento que antropológicamente representa el origen y la plenitud de la vida marcada emocionalmente por la euforia: «Ogni giorno era bello. In cuor la vita/cresceva come vela a mare colmo./Ogni gioia che era in cuor finital ricresceva nel cuor di vita colmo». El acabamiento y renovación de la euforia se produce dentro de un elemento renovable y retornante al infinito (Cuor=mare) que representa el lugar del sentimiento. No solamente el agua figurativiza el ámbito primigenio de una totalidad soñada o la dinamicidad del deseo sino que, como todos los elementos naturales, se antropomorfiza y se convierte en destinataria de la voz del poeta: «Me ne andrei dove il vento mi bacildove il fiume mi parli sommesso ".

El agua del río va asociada desde su infancia a rasgos eufóricos (/dulzura/ y / humedad/) que lo acompañarán en la búsqueda de un elemento arquetípico que lo sumerja en una regresión benéfica: «Non era la città dove la seralebbro cantavo fra le sparse luci/sopra la dolce umidità del fiume./Adesso un biondo sole sulla nera/bottega di mio padre par che brucilla nostra assenza. E non ritrovo il fiume». Pero el rasgo / humedad/va también asociado a una clara alusión (siempre de carácter eufemístico) a actos onanísticos: «Al di là dell' ortaglia ove nell' ombra/i miei atti erano soli/la rotaia deserta luccicava al sole». Precisamente observamos una doble asociación en los vocablos soli-sole, por una parte, atraídos por la iteración fónica paronomásica que nos demuestra el poder de la reflexividad autogeneradora del significante en la poesía de Penna, y por otra parte, al proyectar un sentido ya que el sol (la luz) se opone a la sombra del primer verso (oposición externo/interno) y, además, como veremos, es otro de los elementos arquetípicos que acompañan al objeto de deseo o al deseo con objeto ausente. Este segundo caso es el expresado por los versos anteriores con una alusión sexual que

${ }^{5}$ Stefano Agosti, Cinque analisi. Il testo della poesia, Milano, Feltrinelli, 1983, p. 175

${ }^{6}$ Stranezze, Milano, Garzanti, 1976, p. 103 (libro póstumo). 
volverá a aparecer por contigüedad metonímica en un dístico de Stranezze (p. 114) en ausencia del muchacho amado: «Io muovo incontro al sole/cauto coi miei calzoni ». El narcisismo erótico del adjetivo soli genera paronomásicamente sole, que a su vez, en el sistema poético penniano está ligada al objeto amoroso y en estos versos al autoerotismo.

Sin embargo el agua puede ir asociada a un estado de disforia en el que el acabamiento del deseo coincide con el desbordamiento del agua del río y con la dispersión del seme: /exceso/y /humedad/ son los rasgos dominantes: «Ho puntato la brama in ogni luogo./Sotto la pioggia ho perduto il mio seme./Ora si gonfia il fiume $e$ in me fiorisce/-straripa il fiume- un desiderio nuovo», «Straripa nell' umida notte in silenzio)/il fiume. addio secco vigore della mia gioventù».

Cuando se proyecta la temporalidad la marca de euforia va ligada aún al sol, al pasado feliz (nudi corpi), y la de disforia al río sin agua, al presente que, con el rasgo negativo de /sequedad/ (deserto il fiume), declara la esterilidad del amor: « ̇̇ già lontano il tempolquando vinti al gran sole i nudi corpilturbavano il mio cuore. Adesso brillal deserto il fiume. Ritornato è l'uomolin piedi. Io rido a più sereno amore».

La figurativización del sentimiento amoroso se manifiesta mediante el efecto comparativo con la caída del sol. En Penna el ritmo del imaginario amoroso se acuerda con el ritmo de la naturaleza no sujeto a las leyes de la temporalidad: «Torna un pensier d'amore/nel cuore stanco, come/nel tramonto invernale/ritorna contro il solelilfanciullo alla casa ${ }^{7}$. No es en este caso la naturaleza sino el pensamiento de amor que lo embarga el que se antropomorfiza en una figura concreta que es el objeto de deseo: el fanciullo. La personificación del pensamiento (amore-cuore recoge la rima más antigua de la lírica amorosa) en el muchacho no tiene otra función que la de representar mediante la rêverie el cuerpo de deseo. La unión del fanciullo al disco solar está evidenciada sintagmáticamente por la vecidad de ambos términos (sole-fanciullo) y paradigmáticamente por la simbiosis de ambos en otros poemas en los que el muchacho se integra en la luz solar, es decir, en los que el objeto de deseo se heliofaniza, se funde con el sol: «io nel sole un' umana figura / riguardavo dormire. Indi m'accorsi/che un vero iddio guardava quella forma».

Entre la completa quietud solar con que aparece el joven en estos versos y el dinamismo fugaz de otros poemas fluctúa la oposición interno (estaticidad)/externo (dinamismo) como doble movimiento de la figura del muchacho que forma parte de la realidad y a la vez del sentimiento del narcisismo amoroso del poeta: «Inmobile nel sole la campagna/pareva riascoltare il suo segreto./Un giovane passò ma non so ancora/se

${ }^{7}$ P. V. Mengaldo habra hablado de la relación entre la brevedad de esta poesía -imagen con formas poéticas orientales, como el pantum indonesio (La tradizione del $\mathbf{9 0 0}$, Milano, Feltrinelli, 1975, p. 735), al igual que G. Finzi lo relacionaba con el haikú japonés (Poesta in Italia: Montale, novissimi, postnovissimi, 1959-1978, Milano, Mursia, 1979, pp. 103-104). Por nuestra parte constatamos dicha similitud con poemas amatorios pertenecientes a geografías africanas o asiáticas, para lo cual extraemos algunos ejemplos recopilados por Alfonso M. Di Niola en Canti erotici dei primitivi, Parma, Guanda, 1964, pp. 14 y 28: «Alla fonte la incontrai./Mi diede da bere./La ghermii per il/piccollo collo./ La baciaci a mio piacimiento » « L'ora è pur dolce per l'incontro discretolÈ con l'amico che chiara la pelle». «Bevi birra, o rosso giovanotto $J$ ché ubriachi andrete dalle fanciulle», compárese este último con Ios siguientes versos de Penna: «È bellagiovinezza e basta un pocoldi vino e poi vedete cosa fanno./I miei ragazzi dapprima sì fieri» . 
vero oppure vivo come fiamma/che il sole riassorbiva nel silenzio». La campiña va asociada a dos rasgos, la inmovilidad dentro del sol y la autoauscultación (que le confiere su calidad de narcisismo auditivo). La interioridad del dinamismo externo de la figura del giovane contrasta con la estaticidad de los dos primeros versos, hasta el punto que (y aquí sí interviene la marca del yo: non so) el propio poeta duda de la veraz existencia de aquél, contribuyendo así al carácter fantasmal del objeto de deseo. Si nos detenemos podemos constatar una primera afirmación (Un giovane passò) seguida de una adversativa que no posee, sin embargo, dentro de la cohesión cotextual, un carácter opositivo, puesto que éste se difumina entre dos adjetivos que neutralizan la negación adversativa: vero y vivo. La oposición no se establece entre la existencia/no existencia del paso del joven sino entre los dos adjetivos en relación sinonímica. No se duda del paso del joven sino del hecho de que sea verdadero o vivo, esto es, se duda de una certeza que además queda constatada con el desarrollo de la comparativa del cuarto verso. No solamente la campiña se integra en el sol sino que también el muchacho, convertido en materia solar (fiamma), es absorbido por el sol. El sol (aunador de la luz y del fuego, elemento éste sexualizado masculinamente ${ }^{8}$ ) se convierte en el elemento identificador del fanciullo. Su posición estructural al inicio y al final del poema lo constatan como punto de partida y de llegada. Si, además, se piensa que la gran parábola de la poesía penniana está marcada por el erotismo de la mirada se puede deducir que el centro de esta parábola es el Ojo ubicuo, cuya figurativización no puede ser sino el sol (o la luna ${ }^{9}$ ), ojo avizor por excelencia. Este sol-ojo concentra y funde en sí tanto la actitud narcisista del poeta (impersonalizado en la campiña) como el cuerpo del joven.

El sol, ojo ubicuo que simboliza la plenitud del amor escopofílico, la mirada como única forma posible de amar, está presente en toda aparición y juego del muchacho: «I tuoi calmi spettacoli. La vita./L'amore che li lega. Sole sul colle./E più tardi la luna. aiuto, aiuto!». Si pensamos que el sol es el punto de unión entre el muchacho y el poeta a través de la mirada: «Ma un calmo biondo sole occidentale/versavano i tuoi occhi nel mio viso», «verrà pure il momento/in cui la sole luce/sará negli occhi del fanciullo amico», pero, sobre todo, es el lugar donde mora el fanciullo divino en una especie de ensimismación larvada, podemos observar que el sol representa también el crisol de un narcisismo adolescente que se reproduce en el poeta: «Come dorme il fanciullo/entro di sè nel sole e non ricercalalcuna cosa -io non cerco il fanciullo ». La negación final de la búsqueda del objeto de deseo indica la posesión in absentia del mismo: la transubstanciación del poeta en fanciullo: «Entro l'azzurro intenso di un meriggio d'estateldenso è il fogliame e assorto sotto il lucido sole./Tutto è maturo e pieno. Non sono minacciatelle cose. E nondimeno, lontano come il sole, le vicino, in sè vive -di sè

\footnotetext{
${ }^{8}$ Cfr. «Il fuoco sessualizzato», G. Bacherlard, L'intuizione dell'istante. La psicoanalisi del fuoco, Bari, Dedalo, 1987 (ed. francesa 1967), pp. 167-182.

${ }^{9} \mathrm{La}$ luna va asociada a la mirada en otros poemas: «La luna si nasconde e poi riappare/-lenta vicenda inutilmente mossa/sovra il mio capo stanco di guardare . Es, igual que el sol, un ojo avizor, imperturbable: «Col vespro m' appiatto.IIndi m' accorgolche la luna mi guarda serena». El desdoblamiento de la mirada del poeta en la mirada de la luna produce una mitigación de su afectividad disfórica en la oposición/abatimiento/ -/serenidad/. La luna, como todos los elementos de la naturaleza, tiene una animación basada en lo auditivocontemplativo: «La luna di settembre su la buialvalle addormenta ai contadini il canto» .
} 
solo- il mio amore». El sol preserva las cosas de la corrosión del tiempo y las entroniza en una totalidad benéfica. Ya el adjetivo assorto, aunque referido al follaje, connota el carácter de ensimismamiento narcisista que el sol encierra. La clave nos la dan los dos últimos versos mediante la oposición de los dos adjetivos lontano/vicino, o, lo que es lo mismo, los dos estados interno (narcisismo amoroso)/externo (objeto de deseo-sol).

$\mathrm{El}$ fanciullo penniano en su aspecto onírico se heliofaniza, vive en el sol y se visualiza en la luz. La asociación del muchacho con la luz conlleva una armonización con los elementos primordiales de la naturaleza, cancelando toda huella de temporalidad y de contingencia para acercarse a un estado de totalidad: «Ecco il fanciullo gravido di lucelpiù limpido del verso che lo dice./Dolce stagione di silenzio e solele questa festa di parole in me». «La luce rivedrò, la luce d' orolove brilla il fanciullo. E nella seral brillan, sognando, le sue gote accese».

Si tomamos un ejemplo de la luna avizorante podemos observar cómo es un medio del desdoblamiento del poeta: «La luna ci guardava assai tranquillalal di là dello schermo ov' egli attento/seguiva le indredibili vicende/col suo profilo dibambino, carol a quella luna già, ma assai lontano/solo mezz' ora prima... ». Todo el poema se plantea como un juego de miradas que se entrecruzan y desdoblan. Ya desde el primer momento la luna lanza su mirada hacia el joven y el poeta, los cuales a su vez están mirando una pantalla de cine. Dos deícticos nos confirman esta posición de ambos: al di là, ove, pero el segundo va unido a la tercera persona representada por el muchacho, confirmando que la mirada del poeta se ha trasladado de la luna al joven al describirnos su perfil en un particular de su perspectiva visual. El adjetivo caro comienza el encabalgamiento entre los versos 4-5, y su inclusión en el 4 indica su ligazón visual a bambino como predicativo en aposición, pero el destinatario de esta belleza es la luna y aquí es donde el poema se pone en relación con estructuras míticas. Si recordamos el mito de Endimión sabemos que la luna se enamora de la belleza del joven que duerme eternamente y a él se une. La luna se identifica con el amante (la diosa Selene en la mitología) pero es más importante tener en cuenta el movimiento de la mirada del poeta, primeramente desdoblado en la luna al observar al muchacho y al contemplarse a sí mismo, en segundo lugar al contemplar al chico, y, por último, al unir mirada y afectividad en la luna («profilo di bambino, carola quella luna già»), sólo a través de cuyo ojo iluminante el propio poeta se apodera de la faz de su objeto de deseo. Los dos últimos versos encabalgados nos remiten a la temporalidad y a la aparición epifánica del bambino ya que media hora antes era desconocido. La aparición del objeto de amor es como el ritmo cíclico y regenerativo de la naturaleza, desaparece y vuelve a aparecer. Nos encontramos ante una natura naturans, la naturaleza cuya regeneración es interminable. Esta estrecha relación entre ciertos elementos y el objeto de amor trae consigo la visión de la belleza en aquéllos, cuyo ritmo natural provoca la temporalidad cíclica: «Come è bella la luna di dicembrel che guarda calma tramontare l'anno".

El mester poético de Penna se verifica en dos frentes: por un lado lleva a cabo una reductio sintagmática del poema a través imágenes aisladas en las que se figurativiza el sentimiento de precariedad que lo embarga a causa de la fugacidad del objeto de deseo. Esta fugacidad, que en general, no siempre, va unida a un estado de disforia ( $Q$ Questo prato gid pieno di fanciullo,/pur ne la tramontana al sole vivo,/questa sera, in un' aria 
inumanaldi primavera improvvisa, un mistero.../Cosi vuoto e presente. Io solo vero », "Quando la luce piange sulle strade/vorrei in silenzio un fanciullo abbracciare »), tiene su contrapunto en la estaticidad, cuyo estado, como hemos visto, está ligado íntimamente al sol y a su esencia la luz, y emocionalmente a la euforia. De este estado del muchacho estático en comunión con elementos primordiales como la luz (por asociación del campo semántico, al fuego) y el agua, se apropia el poeta mediante la ensoñación onírica proyectada más allá de lo real o mediante la especularidad de su yo narcisista, condena y salvación de su amor.

Si observamos el poema citado anteriormente: «I tuoi calmi spetaccoli. La vita./ L'amore che li lega. Sole sul colle,/E più tardi la luna. Aiuto, aiuto!», se compone de un léxico de una alta frecuencia de uso y de palabras que poseen un campo semántico muy amplio: spettacoli, sole, luna, vita, amore. Sólo estas palabras sirven al poeta para expresar su estado y su opción sentimental. La concatenación de los versos responde a una sintaxis paratáctica, dividida por pausas señalizadas, en conexión con una semántica que estructura el sentido total del poema. El inicio está marcado por un actante sin función sintáctica definida, en segunda persona, adscribible al fanciullo. El adjetivo calmi enlaza con sole al proyectar en éste el estado de reposo. El sustantivo spettacoli conecta directamente al destinatario implícito (el muchacho) con el poeta que contempla, sujeto virtual: I tuoi calmi spettacoli ${ }^{*}$ vedo. Estos espectáculos son para el poeta la máxima expresión emocional ya que van asociados sintagmáticamente a vita y a amore, que es la causa de unión de algo concreto (spettacoli) a una entidad abstracta y sin límite (vita), que a su vez se identifica con aquél: la parte es el todo y viceversa gracias al espíritu de cohesión del amor ${ }^{10}$, que, además, marca la actividad del yo que ama.

Seguidamente se hace mención a la situación referencial mínima, cuya presencia privilegia esencialmente a la luz, en este caso el sol, y al silencio. En la segunda parte de este verso encontramos un elemento, colle, que, aunque puede representar la idea de elevación hacia el sol y de soledad al ser una elevación solitaria (y no en vano viene a la memoria l'ermo colle de L'infinito de Leopardi), debe su presencia, sobre a todo, a cuestiones fonéticas ya que se produce una paronomasia, una de las tantas manifestaciones de la iteración que jalonan la poesía de Penna ${ }^{11}$. El tercer verso trae de nuevo las dos significaciones observadas del elemento luminoso, la presencia de la luz y la del ojo avizor, pero añade también la coordenada temporal al pasar del día a la noche, señalizado por el adverbio tardi. Observamos pues una gradación: la visibilidad de los gestos del muchacho amado, el reenvío del mismo a la plenitud totalizante, la vida, a través del soplo unificante del amor, marca del yo, y, por último, las coordenadas espaciotemporales. La exclamación final nos lleva a integrarla dentro de la psicología del joven

${ }^{10}$ En otro de sus poemas de carácter gnómico en contra de los moralistas el amor es también el mediador de la armonía del orbe: «Il mondo che vi pare di cateneltutto é tessuto d' armonie profonde». El amor establece una relación entre el fanciullo (lo concreto) y la totalidad del mundo, cuyos elementos primordiales se armonizan gracias a aquél: «Traversare un paese... e li vedere/cheti fanciulli ridestarse a un soffio/di musica e danzare. S'allontanalforma o colore; un sogno. Viva restal la dolce persuasione di una fittalrete d' amore ad inquietare il mondox.

${ }^{11}$ Véase para un elenco detallado de las figuras iterativas en Penna, Giulio Di Fonzo, «L'iterazione», Sandro Penna. La luce e il silenzio, Roma, Edizioni dell'Ateneo, 1980, pp. 39-49. 
para darle un sentido: la llegada de la oscuridad de la noche asusta a los chicos, de ahí la presencia del grito de socorro final, cerrándose el poema con la atención puesta en la figura del muchacho, igual que había iniciado.

El silencio omnipresente que jalona junto a la luz la figura juvenil y el estado emocional del poeta, responde a la imposibilidad de diálogo con el joven, de ahí la reductio operada a nivel de la trama del discurso poético, sea introspectiva o narrativa. No faltan, sin embargo, poemas de aliento descriptivo con la intención de dar cuenta de un sólo hecho: la aparición o desaparición del muchacho. Veamos cómo Penna trata, en un esfuerzo de condensación, de agrupar algunas de sus constantes estilístico-poéticas: «Quando la luce piange sulle strade/vorrei in silenzio un fanciullo abbracciare». Son dos versos en los que está contenida toda la intencionalidad de su autor introducidos por una partícula temporal, que, no obstante, nos sitúa en la intemporalidad y que tiene un matiz de acción indefinidamente repetida, de evento iterativo. Este es uno de los logros intencionados de la poesía penniana: proyectar en la intemporalidad una representación cargada de temporalidad con el mínimo léxico y sin acción narrativa. Inmediatamente la luz ya es un indicio de que antes o después aparecerá la figura a la cual siempre va ligada. Esta figura se sitúa al final de los dos versos para que de esta forma quede fijada en la memoria del lector. La acción personificada de la luz va asociada directamente al rasgo/tristeza/, y, por tanto, a la disforia en el estado de emotividad del poeta, lo cual nos lleva a otras constantes como la personificación (en gran proporción con rasgos del campo semántico amoroso) de los elementos y su relación directa con el estado emocional del poeta, el cual animiza el mundo a imagen y semejanza de su afectividad amorosa. Observamos la rima interna, abundantísima en Penna, a-e (piange-strade) y la aliteración de /ĉ/ (/luce-fanciullo-abbracciare) con el fin de que el nivel fónico contribuya a la iteración léxico-semántica.

La personificación en clave amorosa de la luz tiene además la misión de impersonalizar el estado afectivo del poeta encubierto tras aquélla. La impersonalización penniana está en relación con el carácter eufemístico ${ }^{12}$ y atenuante de una expresión que rehúye del patetismo y de la sensualidad explicitada. La sensualidad existe a priori en esta poesía, de forma que puede permitirse la impersonalización del sentimiento a nivel formal ( Sole senz'ombra su virili corpilabbandonati). Curiosamente en este dístico observamos uno de los pocos verbos activos amorosamente (abbracciare) ya que generalmente dominan las acciones verbales relativas a la mirada. El segundo verso se inicia con un desiderativo que acentúa el carácter hipotético e imposible, en definitiva onírico, de su propio deseo. El silencio, el segundo elemento imprescindible de su universo poético, aparece tras el verbo, tras la marca de la personalización del yo, y nos explica el modo de su opción de deseo. Como hemos apuntado, el silencio puede ser interpretable por la ausencia de diálogo con el muchacho e, incluso, por la misma ausencia constante del objeto de deseo. El poeta asume esta imposibilidad o ausencia en la elección de su propio mutismo, de su imposibilidad de amar con la palabra. Por

${ }^{12}$ Asf lo hacía notar Pasolini en «Una strana gioia di vivere», Paragone, abril 1956, luego en Passione e ideologia, Torino, Einaudie, $1985\left(1960^{1}\right)$, pp. 338-354. 
último, aparece el objeto de deseo y la acción amatoria de abrazarlo, una de las pocas ocasiones en que va más allá de la pura visibilidad.

Si, por una parte, se produce esta reducción, por otra se asiste a una densa iteración en los niveles fónico-léxico, así como a una serie fija de isotopías figurativas y temáticas que constelan toda esta poesía. La aparición (estática/dinámica) del muchacho se corresponde siempre con el amor escopofílico del poeta. Y ya sabemos que dicha aparición va unida casi por completo a una luminosidad que la dota de espacialidad y visibilidad en su más arquetípica esencia, hasta el punto de anteponer la visión de la luz a la del muchacho: «La luce rivedró, la luce d' oro/ove brilla il fanciullo. E nella seral brillan, sognando, le sue gote accese», «Alla finestra ronza col silenzio,/tutto di sole, il cerchio di un fanciullo», «Piove sul campolove incontrai, nel sole, il lieto amico». El objeto de su amor se esfuma como objeto real y se configura como objeto fantasmal integrado en elementos primigenios. El diálogo se establece pues entre el poeta y la naturaleza, y en este diálogo se reconoce la hermandad de Penna con otros poetas como Saba, opuestos a la reproducción de la naturaleza que efectúan otros coetáneos como Ungaretti o Montale.

En Saba existe este diálogo narrativo con la naturaleza de ascendencia romántica. Podemos recordar algunos versos de Nuovi versi alla luna (Trieste e una donna, 19101912) en los que precisamente la representa como un ojo ubicuo, materno: «La luna non mi pare come l'occhioldel sole, l' acceccante occhio che tutto/vede, ma non discerne e non ricorda;/lella sa le presenti e le passate/cose, e per quelle che saranno porta/un finissimo intuito». En realidad este diálogo con la naturaleza es de raigambre leopardiana si se piensa en algunos poemas como Alla luna: «O graziosa luna, io mi ramento,/Che, or volge l' anno, sovra questo collelIo venia pien d' angoscia a rimirarti ", o el Canto notturno di un pastore errante nell' Asia: "Che fai tu, luna, in ciel? Dimmi, che fai,/ Silenziosa luna?». Ante la imposibilidad de respuesta de la naturaleza sólo queda en el aire la pregunta del poeta. En Penna estas preguntas tienen el sabor de la complicidad sentimental ante la fuerza del amor. El amor se alegoriza como la fuerza irresistible que da vida a todos los elementos del universo: «Ditemi, grandi alberi sognanti,/a voi non batte il cuore quando amorelfa cantar la cicala, quando il sole/sorprende e lascia immobile nel tempolil batticuore alla tenera lucertola/perduta fra due mani in un dolce far niente?/Anche a me batte il cuore, e pur non sono/io del fanciulto vittima innocente». La luna como centro de luminosidad es un elemento cómplice para la visibilidad del muchacho, pues no existe amor con la pérdida de la visión de éste: «Nella luce lunare apparve al sommoldel muro del mio harem un ragazzo./Echeggia un colpo efu il silenzio intorno./Non declinare più luna di marzo». Todos los intersticios de la naturaleza están animados y embellecidos por la presencia (real o imaginaria) del objeto de deseo que en muchas ocasiones, como hemos visto, está impersonalizado o pluralizado: "Lumi del cimitero, non mi dite/che la sera d' estate non è bella./E belli sono i bevitori dentrolle lontane osterie», «Livida alba, io sono senza dio./Visi assonnati vanno per le vie/.../Mi abbandono all' amore di quei visi». Tanto bevitori como Visi representan de forma plural e impersonal su verdadero objeto de amor: el fanciullo.

El fanciullo se encuentra diluido e integrado en la naturaleza, por ello cualquier signo de ésta será indicio de la presencia del amor. Naturaleza y amor se identifican y, 
por tanto,éste se manifiesta conla capacidad de renovación que posee la natura naturans. Las apariciones subitáneas de la figura adolescente participan de la «imprevisible» ciclicidad de los fenómenos de la naturaleza que se reiteran indefinidamente en un ritmo incesante de acabamiento y nacimiento. Si el sol del verano había dado su máximo don de belleza al cuerpo juvenil en los poemas de su primer libro Poesie (1939): «Sole senz' ombra su virili corpi/abbandonati. Tace ogni virtù./Lenta l' anima affonda -con il mare-/entro un lucente sonno. D' improvvisolbalzano-giovani isolotti-i sensi», "Il sole che ha brunito questo corpoldi giovinetto cede la sua forza», en los poemas de su libro póstumo Stranezze (1976) implora que el verano, época-símbolo del amor, no lo abandone.

$\mathrm{Si}$, como sabemos, el muchacho está desleído en el sol, y, por extensión, en el aire estivo, la imploración imperativa del poeta está dirigida al mismísimo objeto amoroso, identificándose verano y amor, o, lo que es lo mismo, la figurativización de ambos, luz estival y fanciullo. En el tercer verso hace acto de presencia el amor, no en calidad de sujeto sino de complemento de compañía del verano sobre cuyas espaldas retorna. Tanto el verano, atlante que sostiene el universo amoroso, como el amor, se personifican y se temporalizan con el ritmo de sucesión de las estaciones, aunque el poeta advierta la cercanía del fin de su vida: «Baciami sulla bocca, ultima estate./Dimmi che non andrai tanto lontano./Ritorna con l'amore sulla spalle,led il tuo peso non sarà più vano» ${ }^{13}$. El ciclo de la vida no termina en sí mismo sino que se alarga en el inacabable ciclo de la naturaleza. La vída y el amor, el amante y el amado mueren y renacen, retornan y se reencarnan en otros cuerpos. Este eterno retomo se lleva a cabo con dos de los elementos que han acompañado a lo largo de la vida al muchacho, el sol y el agua. La reencarnación sólo es posible dentro de los elementos primigenios o arquetípicos a través de los cuales se manifiesta el vencimiento del tiempo: «Tu morirai fanciullo ed io ugualmente/ma più belli di te ragazzi ancora/dormiranno nel sole in riva al mare.// Ma non saremo che noi stessi ancora».

La profusión de todas las figuras de repetición (anáfora, epífora, aliteración, repetición de palabras-clave como luce, silenzio, sole...) acentúa la insistencia a nivel consciente e inconsciente de los elementos que lo preservan en su ensoñación erótica frente la realidad hostil. Toda la pasión amorosa de Penna se canaliza a través de la mirada escopofílica que, con la proyección de los elementos naturales alcanzan la figura de deseo, el fanciullo: "Sole con luna, mare con foreste,/Tutt' insieme baciare in una bocca». Encontramos la iteración de dos palabras-clave, sole y mare. Observamos la iteración del fonema /1/: Sole-luna, /b/: baciare-bocca, y del grupo fónico ARE-ORE (mARE-fOREste-baciARE) puesta en funcionamiento por el mecanismo liberatorio del inconsciente.

La transubstanciación del muchacho pone de manifiesto una especie de amor pánico, una reintegración del objeto de deseo en la naturaleza, a través de cuyos elementos el erotismo pudoroso y escopofilico del poeta alcanza su liberación. Únicamente en la naturaleza el fanciullo aparece como objeto de deseo (siempre fantasmáticamente fugitivo) despojado de inhibiciones sociales: «Usci dal verde

${ }^{13}$ Stranezze, op. cit., p. 6. 
inaspettato, ancora/un poco nudo, e subito spari». Pero la mayor simbiosis muchachonaturaleza se realiza en el seno del astro solar, como hemos observado, motor de toda visualización.

La naturaleza no es un ente autónomo como, por ejemplo, acaece en Montale, sino que está supeditada a la ausencia/presencia del joven amado hasta el punto que la desaparición de éste provoca su oscurecimiento y se rompe toda ligazón con una Verdad que pueda erigirse en un centro: "Avete mai provato, in un'aria serenale in un paese puro, -ma però non vi date/sentimento di sorta,- a guardar fissamenteld' improvviso un ragazzo? L'innocenzalforse risalirà con la sua biciclettalla lenta strada, e poi vi sarà toltald' un tratto dalla polvere di un camion./Quando poi schiarirà, cercate ancora/ sulla strada, o nel cuore, il vuoto incanto./Fingerà la natura un suo tramonto./E tutto vi parrà -ma non vi date sentimento di sorta-falso e vero". La naturaleza cambia en función de la vivencia amorosa, adquiere un rol relacional entre los elementos que la componen. No existe la causa aislada sino una verdadera red tupida de relaciones armónicas que la convierten en una fuerza benéfica y fecundadora simbolizada por la flor como elemento de relación sexual por excelencia: «Non è la costruzione il lieto donoldella natura. Un fiore chiama l'altro" (recordemos: "Viva restalla dolce persuasione di una fitta/rete d'amore ad inquietare il mondo», "Il mondo che Vi pare di catene/tutto è tessuto d' armonie profonde»).

La antropomorfización del sol es también una forma de totalizar el amor hacia el muchacho puesto que aquél como punto máximo de visibilidad y de contacto con el joven representa el propio deseo (siempre escopofilico) amoroso del poeta: «Era il settembre. Riandava la gente/rumorosa alle strade. IIl sole amava/il vino el'operaio» . El sol es siempre un elemento marcado benéficamente por su relación privilegiada con el fanciullo y por su pertenencia al reino arquetípico de la luz, a través de la cual es posible la visión, única forma, según hemos visto, de amar para Penna. Por ello mismo el imaginario de la luz es el ámbito mítico predilecto para un reposo eterno, de forma que quietud y luz se unen de nuevo no bajo el signo del amor sino de la muerte: «Io sono in un locale greve e nero,/pieno di fumo, pieno di parole./Ma sono assente: e sogno il cimitero/di un piccolo villaggio sotto il sole ${ }^{14}$, que no es sino el inicio de otra nueva vida con la que cierra su poemario Stranezze (p. 126) en el que invoca al dios del amor: «Un altro mondo si dischiude: un sognolfanciulla mia beta sotto il sole/medesimo (oh gli antichile dorati fanciullo). Un lieve sogno/la vita.../Ricordati di me dio dell' amore».

El amor es finalmente un ente divino, pero el sol continúa imperturbable e idéntico: «sole/medesino». 